\title{
Le (dé)goût d'un printemps : la construction sociale de la violence et de l'extrémisme politique lors du conflit étudiant québécois
}

\section{Gabriel Blouin Genest}

\section{(2) OpenEdition \\ Journals}

Édition électronique

URL : http://journals.openedition.org/conflits/18507

DOI : 10.4000/conflits. 18507

ISSN : $1777-5345$

Éditeur :

CCLS - Centre d'études sur les conflits lilberté et sécurité, L'Harmattan

Édition imprimée

Date de publication : 26 décembre 2012

Pagination : 160-166

ISBN : 978-2-336-00248-4

ISSN : 1157-996X

Référence électronique

Gabriel Blouin Genest, « Le (dé)goût d'un printemps : la construction sociale de la violence et de l'extrémisme politique lors du conflit étudiant québécois », Cultures \& Conflits [En ligne], 87 | Automne 2012, mis en ligne le 26 décembre 2012, consulté le 30 mars 2021. URL : http://

journals.openedition.org/conflits/18507; DOI : https://doi.org/10.4000/conflits.18507 


\title{
Le (dé)goût d'un printemps : la construction sociale de la violence et de l'extrémisme politique lors du conflit étudiant québécois
}

\section{Gabriel BLOUIN GENEST \\ Gabriel Blouin Genest est docteur en études politiques et professeur à temps partiel à l'Université d'Ottawa. \\ Violence et construit social : inventaire sémantique de la production d'un extrémisme politique au Québec}

\begin{abstract}
A lors que le conflit social et étudiant québécois vient tout juste de prendre - fin, avec l'élection d'un gouvernement du Parti québécois ${ }^{1}$ le 4 septembre 2012 et l'abolition de la hausse des frais de scolarité qui avait provoqué la crise étudiante au Québec, les mois passés nous permettent tout de même d'y porter un regard analytique, en s'intéressant notamment à la « cartographie langagière » qui a marqué ce conflit. Ce mouvement de protestation des étudiants québécois, initié en réaction à une augmentation des droits de scolarité de $75 \%$ sur cinq ans, a mené à la plus longue grève de l'histoire du Québec. Pour forcer le retour en classe, le gouvernement de Jean Charest avait voté le projet de loi 78 (devenue par la suite loi 12) qui suspendait la session pour les étudiants en grève et limitait substantiellement le droit de grève et de manifestation, sous peine de pénalités majeures pour les individus et fédérations étudiantes. C'est dans ce contexte que le gouvernement en place a déployé une habile production langagière associant étudiant et violence. Retour sur ces narratifs qui donnent un si mauvais goût au «printemps québécois ».
\end{abstract}

Ces narratifs ont (re)produit une forme d'extrémisme politique sur lequel sont venus se greffer les différents protagonistes de la crise étudiante, façonnant une lubie idéologique qui n'a de sens que dans les termes utilisés pour la caractériser. Les forces représentationnelles en place, polarisées autour d'une

1. Qui a remplacé le gouvernement libéral de Jean Charest. 
syntaxe de l'extrémisme et de la violence, ont engendré cette tendance dualisante qui a été si bien instrumentalisée durant le conflit étudiant par le gouvernement libéral de Jean Charest : les étudiants et leurs " carrés rouges » 2 ont ainsi été érigés en symboles de la violence, de l'intimidation, de l'extrémisme et du radicalisme. Jean Charest avait dès le début posé en ces termes le débat social qui s'installait entre son gouvernement et les étudiants. La première condition qu'il avait imposée pour la négociation était que tous les leaders étudiants condamnent officiellement la violence - celle des étudiants, évidemment, non pas celle des forces policières ou du gouvernement. Cette condition ne visait qu’à associer, par la force des mots et du discours, le mouvement étudiant à certains actes de vandalisme qui avaient marqué les manifestations, tout en permettant au gouvernement de ne pas s'investir honnêtement et de manière transparente dans un processus de négociation avec les fédérations étudiantes. Ce que cherchait à faire le gouvernement, c'était précisément de lier linguistiquement violence, extrémisme et mobilisation étudiante. Les « carrés rouges » ont ainsi été directement associés à la violence et à l'intimidation. Ainsi, la ministre de la Culture Christine St-Pierre a pu déclarer, en parlant du chanteur Fred Pellerin qui, dans le contexte de crise sociale, avait refusé d'être fait chevalier de l'Ordre national du Québec : « il a le droit de porter le carré rouge, on est dans la liberté d'expression, mais nous, on sait ce que ça veut dire le carré rouge, ça veut dire l'intimidation, la violence $[. . .]^{3}$ ». Jean Charest a quant à lui dénoncé la «banalisation de la violence » qu'il percevait, soulignant que « [l]e discours banalisant la violence et l'intimidation s’impose petit à petit au Québec [...]. L'erreur, c'est de ne pas réagir, c'est de ne rien dire, de laisser passer. Depuis des mois, ça avance d'un pas à l'autre, on tolère l'intimidation, la violence $[. . .]^{4}$ ». Par un ironique retournement de sens, le Premier ministre a même réussi à poser son propre gouvernement en victime, en opprimé d'une classe étudiante sans foi ni loi : «[c]es gens-là pensentils qu'on n'a pas de famille, qu'on n'est pas des êtres humains ? C'est comme si on vient déshumaniser la politique, déshumaniser la vie ${ }^{5}$ ». Comme si les 2739 arrestations (nombres provisoires), les dizaines de blessés, les ecchymoses, les yeux crevés, les dents cassées, la répression judiciaire et financière ou la discrimination à l'œuvre dans ce conflit n'avaient aucune emprise sur le sens et la matérialité du mot violence ${ }^{6}$. Cette construction sociale de la violence a été habilement associée non pas uniquement aux partis d'opposition,

2. Le carré rouge est le symbole de la mobilisation étudiante. Il s'oppose entre autres aux carrés verts (pour la hausse) et aux carrés blancs (pour une trêve entre le gouvernement et les étudiants).

3. Voir Nadaud J-F., «Le carré rouge de Fred Pellerin : “violence et intimidation”, affirme la ministre de la Culture », Le Devoir, 9 juin 2012 (www.ledevoir.com/culture/actualites-culturelles/352046/le-carre-rouge-de-fred-pellerin-violence-et-intimidation-affirme-la-ministrede-la-culture, consulté le 12 juin 2012).

4. Voir Robitaille A., Porter I., «Charest dénonce une fois de plus la "banalisation de la violence” ", Le Devoir, 14 juin 2012 (http://m.ledevoir.com/politique/quebec/352455/jean-charest-sur-la-violence, consulté le 16 juin 2012).

5. Ibid.

6. Cette violence du gouvernement et des forces policières a même amené le rapporteur spécial 
ce qui est classique, mais aussi et surtout à l'extrémisme et au radicalisme politique. Pour une nation qui a toujours craint les conflits ouverts et l'opposition directe, le radicalisme est une chimère qui, sans l'ombre d'un doute, montre l'importance qu'a pris, et qu'aura dans l'histoire, ce conflit. Lors du Grand Prix automobile de Montréal organisé début juillet 2012, et devant les risques attendus de perturbations, Jean Charest a par exemple brandi la menace posée par de soi-disant « groupes d'extrême gauche 7 » s'opposant au capitalisme et à la marchandisation des corps. Le ministre des Finances Raymond Bachand en a profité pour accuser cette extrême gauche d'user de pression : « [j]e ne suis pas du genre à reculer devant l'intimidation [...]. Car le jour où on commence à reculer devant l'intimidation, ce jour-là la démocratie est morte 8 ». Martin Lemay, député du principal parti d'opposition (le Parti québécois), a également ouvertement affiché sa «crainte de voir les mouvements d'extrême gauche subordonner la lutte pour l'indépendance du Québec à la révolution communiste ${ }^{9}$. Oui, la révolution communiste. Un haut fonctionnaire du ministère des Affaires municipales du Québec a même suggéré, dans une lettre ouverte envoyée au quotidien Le Soleil, que face à ce péril estudiantin,

" il faut s'organiser pour reconquérir le terrain. Dans les années 1920 et 1930, c'est ce qu'on [sic] fait les mouvements fascistes, en appliquant aux gauchistes leur propre médecine. Ceux-ci en ont gardé un souvenir si cuisant que, trois quarts de siècle plus tard, ils s'acharnent encore à démoniser cette réaction de salubrité politique. Les opposants aux grèves doivent donc cabaler, s'organiser pour franchir en masse les lignes de piquetage, apostropher les porteurs de carrés rouges où qu'ils les rencontrent, répondre à l'intimidation par le défi $10 »$.

des Nations Unies sur le droit à la liberté de réunion et d'association pacifiques, Maina Kiai, ainsi que la section canadienne francophone d'Amnistie internationale à demander au gouvernement du Québec de faire la lumière sur divers évènements ayant potentiellement entravé plusieurs droits de l'homme et du citoyen. Le gouvernement n'a offert pour toute réponse que sa « surprise » de voir l'ONU et certaines ONG s'intéresser au Québec tandis que d'autres crises sont à l'œuvre ailleurs dans le monde.

7. Voir Ouellet M., Larocque S., "Charest défend les interventions contre les "groupes d'extrême gauche” ", La Presse, www.lapresse.ca/actualites/quebec-canada/politique-quebecoise/201206/11/01-4533862-charest-defend-les-interventions-contre-les-groupes-dextreme-gauche.php?utm_categorieinterne $=$ trafficdrivers\&utm_contenuinterne $=$ cyberpresse_vous_suggere_4533455_article_POS2, consulté le 16 juin 2012).

8. Voir «Conflit étudiant - Le gouvernement ne cédera pas, dit Bachand », Le Devoir, 11 juin 2012 (www.ledevoir.com/politique/quebec/352162/le-gouvernement-ne-cedera-pas-ditbachand, consulté le 21 juin 2012).

9. Voir Lemay M., "Lettre à un jeune gauchiste - 1ère partie », La Métropole, 19 avril 2012 (www.lametropole.com/blog/martin-lemay/lettre-à-un-jeune-gauchiste-1ère-partie, consulté le 19 juin 2012).

10. Nos italiques. Legault J., "Qui dénoncera qui cette fois », Voir Montréal, 16 avril 2012 (http://voir.ca/josee-legault/2012/04/16/qui-denoncera-qui-cette-fois/, consulté le 18 juin 2012). 
Stéphane Gendron, maire de la ville d'Huntingdon, a même réclamé « [1]'armée et la bastonnade 11 » pour venir à bout des manifestants. Cette production d'un Autre dangereux, violent, a amené la division sur la menace extrémiste de la Sureté du Québec, la police nationale, à interroger pendant plusieurs heures Gabriel Nadeau-Dubois, un des quatre principaux leaders étudiants. On s'est ainsi retrouvé dans un véritable délire identitaire et représentationnel qui, à force de propagande et de stratégie de communication bien ficelée, a transformé des groupes sociaux réclamant l'accès à une éducation abordable, une équité intergénérationnelle et une égalité des chances en groupes radicaux et violents menaçant la paix sociale.

\section{Une révolution qui tourne carré : profilage, discrimination et architecture répressive pour les « carrés rouges"}

Cette construction de l'extrémisme politique, d'un Autre dangereux et menaçant a été reprise et reproduite sans ménagement par un ensemble de commentateurs politiques et de média, polarisant encore plus la population autour de cette dichotomie identitaire opposant sans nuance ordre et extrémisme politique. Les « carrés rouges » se sont ainsi retrouvés porte-étendards d'une révolution violente, d'un extrémisme politique oppressant aux relents de communisme et de révolution bolchévique. Denise Bombardier, une personnalité publique et médiatique d'importance au Québec, a magistralement fait écho à cette construction en spécifiant que le conflit social actuel était l'œuvre d'une « [...] extrême gauche longtemps souterraine et de ce fait hyperactive, pour qui le noyautage, l'infiltration et l'intoxication selon les meilleures références soviétiques d'avant l'effondrement du mur de Berlin, servent de praxis. Des groupuscules anarchiques, anticapitalistes qui radotent sur un Cuba du nord $[\ldots]^{12}$ ». Comment cette caricature d'un Autre dangereux, d'un ennemi interne menaçant la stabilité et l'ordre social, peut-elle mener à autre chose qu'à un profilage et une discrimination généralisés ainsi qu'à une stigmatisation sociale au parfum historique nauséabond ? Un article publié dans Le Devoir et pour lequel deux journalistes ont décidé de porter le carré rouge révèle avec force cette stigmatisation. Leur épopée dans le métro de Montréal lors du Grand Prix de 2012 est symptomatique du processus de profilage et de discrimination à l'œuvre aujourd'hui : on a ainsi arrêté et fouillé systématiquement tous ceux qui portaient le carré rouge, incluant les deux journalistes en question. Lorsque l'une d'entre elles leur demande la raison de cette fouille, un des policiers répond tout bonnement « Parce que vous arborez un signe révolutionnaire 13 ». Révolutionnaire ? Mais selon qui ? Devrait-il être laissé

11. Voir Lapierre J., «Mouvement étudiant : appuis et oppositions en ligne », Radio-Canada.ca, 21 mars 2012 (http://blogues.radio-canada.ca/surleweb/2012/03/21/mouvement-etudiantappuis-et-opposition-en-ligne/, consulté le 21 juin 2012).

12. Voir Marc-André Cyr, «La grande peur rouge ", Voir Montréal, 28 Mai 2012. Voir : http://voir.ca/marc-andre-cyr/2012/05/28/la-grande-peur-rouge/ Page consultée le 19 juin 2012.

13. Voir http://m.ledevoir.com/societe/actualites-en-societe/352102/recit-d-un-petit-voyage- 
aux services de police le loisir de définir la légitimité des mouvements de contestation sociale et leur caractère révolutionnaire ? C’est précisément ce que permet la loi 12 adoptée par le gouvernement Charest pour mater la révolte et forcer le retour en classe des étudiants en grève. Plusieurs entreprises, publiques comme privées, ont même interdit le port du carré rouge, forçant les employés à taire leurs prises de position. La Commission des droits de la personne et de la jeunesse ainsi que la Ligue des droits et liberté du Québec ont affirmé avoir reçu de nombreuses plaintes de citoyens ayant vécu une situation de discrimination en raison du port du carré rouge ${ }^{14}$.

\section{Québec, la Belle Province ? Les mots du « printemps québécois » et de la grève étudiante}

Sur le site touristique officiel du gouvernement du Québec, nous pouvons lire que notre province " constitue un lieu unique en terre d'Amérique. Unique en raison de sa géographie, de son histoire et de sa culture, qui ont forgé son identité teintée d'effervescence et d'audace. Le Québec, c'est une cordialité toute simple, une authenticité sans compromis et l'intensité à portée de main ! 15 ». Le Québec a toujours cherché à construire - et à reproduire cette image de bon vivant, cet authentique " homme du Nord » moderne, enraciné dans ses grands espaces, toujours simple, humble, presque apolitique malgré les turpitudes de l'indépendantisme. Ici, les révolutions sont « tranquilles 16 et les génocides ${ }^{17}$ sont tus. C'est notre construction identitaire. C'est ce que nous voulons être, et c'est ce que l'on projette de nous, dans un désir d'émerveillement qui est autant destiné aux Autres qu'à nous-mêmes. Cette identité se fait et se refait au gré des évènements qui marquent le contexte social et politique du Québec. Le «printemps québécois » et la grève étudiante de 2012 font décidément partie de ces moments. Ce qui est toutefois particulier ici, c'est à quel point nous avons délaissé nos représentations identitaires habituelles pour nous transposer vers une construction sociale de la violence et de l'extrémisme dont nous a peu habitué l'espace politique au Québec. Le gouvernement Charest s'est ainsi employé à édifier une surprenante construction sociale identitaire du mouvement étudiant, construction sociale s'appuyant sur une structure langagière reproduisant, voire dépassant, une rhétorique gouvernementale associant manifestations étudiantes et violence. Comme le soulignait Jean-Pierre Lorange dans les pages du Monde 18,

en-metro-avec-un-carre-rouge, consulté le 21 juin 2012.

14. Voir http://www.radio-canada.ca/nouvelles/societe/2012/06/21/006-plaintes-droits-personne-carre-rouge.shtml Page consultée le 24 juin 2012.

15. Voir http://www.bonjourquebec.com/fr-fr/lequebec.html Page consultée le 22 juin 2012.

16. En référence à la Révolution tranquille, période historique concernant principalement la décennie 1960 et ayant entre autres permis au Québec de mettre en place les bases de l'État providence, de soutenir l'émergence d'un nouveau nationalisme québécois et de concrétiser la séparation de l'État et de l'église tout en favorisant un développement économique national.

17. En référence à l'extermination et l'asservissement des premières nations présentes bien avant l'arrivée des colons français et anglais en terre d'Amérique, et dont on peine toujours à reconnaître l'existence historique. 
c'est non seulement à la nouvelle génération de citoyennes et citoyens que s'en est pris le gouvernement québécois, mais également, et avant tout, au sens des mots, à leur valeur. Violence, extrémisme, intimidation, radicalisme sont autant d'expressions dont fut affublé le mouvement social et étudiant lors de la crise de 2012. La société québécoise s'est ainsi polarisée autour d'une sémantique de la peur et de l'angoisse.

Cette crise étudiante est venue nous rappeler l'impact des mots, des termes, du vocable utilisés lors des conflits sociaux. Le langage, dans ce contexte, transporte, transpose, transforme des réalités et des identités. En apparence périphériques, le discours et les narratifs ne participent pas moins de la (re)production de ce qui constitue le fond, la substance, en constituant l'espace symbolique où se fait l'acquisition du sens. Le conflit étudiant au Québec l'a montré, la crise ne s'est pas jouée dans la rue, elle n'a pas opposé forces de l'ordre et gouvernement aux manifestants. La crise s'est jouée au niveau des mots et de leurs significations, au niveau de la construction sociale de la violence et de l'extrémisme qui ont été associés aux étudiants. Cette caractérisation des acteurs est inséparable de la langue qui la porte, la fait vivre et la structure. Le langage utilisé dans cette crise, en s'inscrivant dans cet espace sociolinguistique de formation et d'appropriation du sens, a acquis une fonction fondamentale dans la construction de la réalité perçue de ses différents acteurs. L'acte d'élocution, de caractérisation de ce que sont les étudiants et leurs supporteurs est devenu dans ce contexte action, fonction de définition de ce qui est. Il est devenu la condition même d'entendement et d'émergence de ces objets sociolinguistiques que sont les « étudiants », les « manifestants », la « violence » ou « l'extrémisme ». Problématiser l'impact des mots, les concevoir comme un système hautement organisé et encodé permettant la représentation et la construction des identités ${ }^{19}$, c'est souligner la manière dont ils sont, en eux-mêmes, impliqués dans la possibilité d'expression ou non de certains objets et sujets. La langue, pour reprendre une formulation derridienne, y devient une série de signes, et le sens qui en est tiré s'établit non pas selon l'essence de la chose, mais bien par une suite de juxtapositions où la chose est mise en valeur par rapport à son opposé, ce à quoi elle peut se distinguer. Dans cette perspective, il faut rendre au langage son caractère social et politique, sa capacité à définir, dans l'espace social, ce qui est, mais également ce qui n'est pas. Il s'agit ici de la resituer dans ce système hautement instable de signes et de représentations qui participe à la genèse du sens par l'intermédiaire d'une construction simultanée de l'identité et de la différence ${ }^{20}$. La violence par rapport à la paix et l'ordre. L'extrémisme par rapport à la modération et au centrisme. La turbulence des étudiants par rapport à l'autorité du gouvernement.

18. Voir http://www.lemonde.fr/idees/article/2012/06/15/le-sens-retourne-des-mots_ 1718838_3232.html Page consultée le 19 juin 2012.

19. Saïd E.W., L'Orientalisme. L'Orient créé par l'Occident, Paris, Seuil, 2005, p. 21.

20. Hansen L., Security as Practice: Discourse Analysis and the Bosnian War, Londres, Routledge, 2006, p. 17. 
La clameur de la rue face à la démocratie des institutions. Les mots ne doivent donc pas être ici entendus uniquement en tant qu'artifices techniques de locution, mais aussi comme codes et conventions collectivement partagés qui structurent le couple conceptuel inclusion/exclusion et qui permettent l'établissement d'une structure dynamique de reproduction d'identités et de subjectivités particulières. Cette production identitaire passe par des pratiques ou forces représentationnelles fixant les identités dans des systèmes de signification qui les mettent en opposition les unes par rapport aux autres, piégeant l'énonciateur et son objet dans une relation inéluctablement oppositionnelle avec ce qui les entoure. Cette inclination à penser en termes dichotomiques facilite ainsi la production d'espaces d'altérité et de mutisme dans lesquels l'énonciation et l'atteinte du statut de locuteur sont restreintes ou limitées pour certaines identités et, en même temps, facilitées pour d'autres. L'importance du lieu d'élocution prend ici tout son sens, surtout lorsque celui-ci se situe au cœur du politique et de l'architecture sécuritaire de l'État.

C'est donc l'ensemble des structures policières, judiciaires, législatives, mais aussi identitaires et sociales qu'a mobilisé l'appareil politique québécois pour mettre fin à la contestation étudiante et sociale. Avec la loi 12, le gouvernement a su créer un état d'exception schmittien dans lequel les mécanismes de gouvernance ont été mis à profit d'une catégorisation dichotomique du bien et du mal, de ce qui est légitime en société démocratique et de ce qui ne l'est pas, du droit et des sans droits. Le Québec s'est ainsi institué en une sorte d'idéal foucaldien de société du contrôle et de la surveillance. Le Québec, "province pestiférée » ? Nul doute, avec le "printemps québécois », c'est bel et bien à la structuration d'une société disciplinaire sur le modèle de la «ville pestiférée » dont parlait Michel Foucault auquel nous avons assisté. Idéal architectural de surveillance, de contrôle, de correction, de répression, de stigmatisation, d'organisation des corps. Dispositif de sécurité inextricable du libéralisme comme mode de gouvernance. Voir sans être vu, en catégorisant les individus. Les monter les uns contre les autres. Les forcer à s'opposer. Carré rouge, carré vert, carré blanc, carré néant. Vous avez le goût d'un « printemps québécois " ? Le désir d'une douce occidentalisation du printemps arabe ? Nous en avons le dégoût. 